\title{
Ultrastructural analysis of chromatin in meiosis I + II of rye (Secale cereale L.)
}

\author{
J.F. Zoller, U. Hohmann, R.G. Herrmann, and G. Wanner \\ Department Biologie I, Bereich Botanik, Ludwig-Maximilians-Universität München, München (Germany)
}

\begin{abstract}
Scanning electron microscopy (SEM) proves to be an appropriate technique for imaging chromatin organization in meiosis I and II of rye (Secale cereale) down to a resolution of a few nanometers. It could be shown for the first time that organization of basic structural elements (coiled and parallel fibers, chromomeres) changes dramatically during the progression to metaphase I and II. Controlled loosening with proteinase $\mathrm{K}$ (after fixation with glutaraldehyde) provides an enhanced insight into chromosome architecture even of highly condensed stages of meiosis. By selective staining with platinum blue, DNA content and distribution can be visualized within compact chromosomes as well as in a complex arrangement of fibers. Chromatin interconnecting threads, which are typically
\end{abstract}

observed in prophase I between homologous and non-homologous chromosomes, stain clearly for DNA. In zygotene transversion of chromatid strands to their homologous counterparts becomes evident. In pachytene segments of synapsed and nonsynapsed homologs alternate. At synapsed regions pairing is so intimate that homologous chromosomes form one filament of structural entity. Chiasmata are characterized by chromatid strands which traverse from one homolog to its counterpart. Bivalents are characteristically fused at their telomeric regions. In metaphase I and II there is no structural evidence for primary and secondary constrictions.

Copyright @ 2003 S. Karger AG, Basel
Meiosis is characterized by two separate processes: meiosis I when recombination takes place and new recombined homologous chromosomes segregate, and meiosis II, when sister chromatids are separated in a process which is regarded to be analogous to mitosis. Prophase of meiosis I is so complex that it is divided into five substages - each characterized by a specific chromosome conformation. In leptotene, chromosomes are ordered as a bouquet, with telomeres attached to a restricted region of the nuclear membrane, which might facilitate recognition and alignment of homologs (Bass et al., 2000; Scherthan, 2001). In zygotene when homologs associate, synapsed and non-synapsed homologous chromosomal segments are lying

\footnotetext{
Supported by the Deutsche Forschungsgemeinschaft (SFB-TR1, Ho1217/2) and the Fonds der Chemischen Industrie.

Received 18 August 2003; revision accepted 3 December 2003.

Request reprints from: Jutta F. Zoller, Botanisches Institut der LMU Menzinger Strasse 67, D-80638 München (Germany) telephone: +49-89-17861-191; fax: +49-89-17861-248 e-mail: zoller@botanik.biologie.uni-muenchen.de

Present address of U. Hohmann:

Institut für Pflanzenbau und Pflanzenzüchtung der

Christian-Albrechts-Universität zu Kiel

Olshausenstrasse 40, D-24098 Kiel (Germany)
}

apart. In pachytene, synapsis is completed and recombination takes place within a specialized recombination structure - the synaptonemal complex (SC). During diplotene, separation of homologs starts. Chiasmata, which become visible at distal regions of the chromosomes in diakinesis, are regarded as relicts of crossing-over events (von Wettstein et al., 1984). In metaphase I bivalents are ordered in the metaphase plate providing a correct segregation of homologous chromosomes.

Within the past decade recombination has been extensively studied by molecular and light microscopic (LM) analysis of different eukaryotes (mammals, amphibians, insects, yeast and plants; Sym et al., 1993; Scherthan et al., 1994; Vazquez et al., 2002; Crackower et al., 2003). These investigations were predominantly focused on chromosome behavior and synapsis of homologs. Despite the fact that plants are attractive models for investigation of chromosome pairing, only minor information is available about chromatin ultrastructure during meiosis I and II. Most investigations were focused on the SC by light microscopy (LM) or transmission electron microscopy (TEM). Scanning electron microscopy (SEM) has proven to be a valuable tool for structural investigations of mitotic chromosomes (Nagai et al., 1982; Murayama, 1983; Wanner et al., 1991; Martin et al., 1996; Iwano et al., 1997; Zoller et al., 2004). It was shown that the common feature of human and plant mitotic chromosomes (Martin et al., 1996) are basic substructures of

\footnotetext{
KARGER Fax +41613061234 E-mail karger@karger.ch www.karger.com

(C) 2004 S. Karger AG, Basel 0301-0171/04/1051-0145\$21.00/0
}

Accessible online at: www. karger.com/cgr 
10-15-nm fibers and 30-nm fibers (Nagai et al., 1982; Utsumi, 1982, 1985; Hanks et al., 1983; Murayama, 1983; Wanner and Formanek, 2000). The 10-nm fiber - also designated elementary fibril - represents the first level of compaction of DNA by a nucleosomal array (Woodcock, 1973; Olins and Olins, 1974; Woodcock et al., 1976). The 30-nm fiber represents the second level of compaction, which is discussed to be a solenoidal arrangement of the elementary fibril (Thoma et al., 1979). This fiber class can be predominantly observed in mitosis to be arranged either parallel or aggregated into chromomeres (Reznik et al., 1991; Wanner et al., 1991; Martin et al., 1996; Iwano et al., 1997; Wanner and Formanek, 2000).

Although chromosome ultrastructure in mitosis has been analyzed in detail, only minor information is available about the arrangement of chromatin in meiosis. There are only few ultrastructural investigations on meiotic chromosomes (Sumner, 1986; Barlow et al., 1993; Barlow, 1996; Inaga et al., 2000, 2002). This could be due to a lack of an appropriate technique for preparation of meiotic chromosomes for SEM and, in addition, be complicated by the high compaction of meiotic chromatin (about twice as much as in mitosis), which prevents investigation of (three-dimensional) chromosome ultrastructure (Zoller et al., 2004). We have modified the drop-cryo technique, developed for mitotic chromosomes by Martin et al. (1994), which allows investigation of all stages of meiosis I and II in routine (Zoller et al., 2004). The major focus of the present investigation is a detailed SEM analysis of meiosis-specific pairing structures: synapsis in zygotene, recombination structures in pachytene and diplotene, and chiasmata in diakinesis. As meiotic chromosomes are highly condensed, (glutaraldehyde-fixed) chromosomes were gradually loosened with proteinase $\mathrm{K}$ to get a better insight into chromosome architecture (Wanner and Formanek, 2000). Staining with platinum blue (Wanner and Formanek, 1995) was applied to provide additional information about DNA distribution within chromosomes and the nature of structures investigated.

\section{Materials and methods}

Plant material and chromosome drop-cryo preparation

The spikes of rye (Secale cereale L.) cv. Sorom were harvested and fixed in ethanol:acetic acid $(3: 1, \mathrm{v} / \mathrm{v})$ before anthers of each floret were dissected and stored for at least 2 days at $-20^{\circ} \mathrm{C}$. The developmental gradient of pollen mother cells within anthers of each spike was examined in the phase-contrast microscope. The chromosome drop-cryo preparations were made according to Martin et al. (1994) with the following modifications: anthers were digested with $1.25 \%$ cellulase ("Onozuka R10"; Sigma Chemicals, Munich, Germany) and $1.25 \%$ pectolyase ("Pectolyase Y-23"; Kikkoman, Japan). For hypotonic treatment cell suspensions were incubated in $75 \mathrm{mM} \mathrm{KCl}$ and washed several times with $70 \%$ ethanol before final fixation with 3:1 ethanol: acetic acid $(v / v)$. Suspensions were dropped onto laser-marked glass slides (LaserMarking, Fischen, Germany). Before chromosome spreads dried, a drop of $45 \%$ acetic acid was added and slides were frozen on dry ice. After removal of the coverslip, chromosomes were fixed with $2.5 \%$ glutaraldehyde (buffered with $75 \mathrm{mM}$ cacodylate, $2 \mathrm{mM} \mathrm{MgCl}_{2}, \mathrm{pH}$ 7.0).

\section{Proteinase K treatment}

For proteinase $\mathrm{K}$ treatment, chromosome spreads were fixed with $2.5 \%$ glutaraldehyde (buffered with $75 \mathrm{mM}$ cacodylate, $2 \mathrm{mM} \mathrm{MgCl}_{2}, \mathrm{pH} 7.0$ ) before being treated with proteinase $\mathrm{K}(1 \mathrm{mg} / \mathrm{ml}$ which equals $35.5 \mathrm{mAnsons}$ units $/ \mathrm{ml}$, ICN Biochemicals) for $30 \mathrm{~min}$ at $37^{\circ} \mathrm{C}$. The slides were washed in aqua dest. and dehydrated in an acetone series.
DNA staining

Platinum blue was prepared according to Wanner and Formanek (1995). The specimens were washed in cacodylate buffer $(75 \mathrm{mM}$ cacodylate, $2 \mathrm{mM}$ $\mathrm{MgCl}_{2}, \mathrm{pH}$ 7.0) and aqua dest. before incubation with a solution of platinum dye under a coverslip for at least $30 \mathrm{~min}$ at room temperature. The slides were washed in aqua dest. before dehydration in an acetone series.

Preparation for scanning electron microscopy

The samples were washed in cacodylate buffer and aqua dest. before dehydration in an acetone series $(20,40,60,80$ and $100 \%$ acetone) and critical point dried with liquid $\mathrm{CO}_{2}$ or dried by using HMDS (hexamethyldisilazane; Sigma, Germany). The specimens were sputter-coated with approximately $3 \mathrm{~nm}$ platinum with a magnetron sputter coater (BalTec), and for BSE detection with about $3 \mathrm{~nm}$ of carbon by evaporation (BalTec). Specimens were examined with a Hitachi S-4100 field-emission scanning electron microscope operated either at $5-8 \mathrm{kV}$ for SE images or at $15 \mathrm{kV}$ for simultaneous detection of secondary electrons (SE) and back-scattered electrons (BSE). SEM images were recorded either with black and white negative film or with Digiscan ${ }^{\mathrm{TM}}$ hardware and processed with Digital Micrograph 3.4.4 software (Gatan Digital Micrograph, Inc., Pleasantdon, CA, USA).

\section{Results}

\section{Chromosome drop preparation}

The modified "drop-cryo" preparation technique proved to be suitable for the isolation of chromosomes of pollen mother cells of all stages of meiosis I and II. Contamination of chromosomes with cytoplasmatic material was negligible. Chromatin was structurally well preserved and provided insight into details of chromatin elements down to $10 \mathrm{~nm}$ in diameter. There are two dominant classes of fibers, a population of rather smooth "10-15-nm fibers" and a population of "30-nm fibers" frequently exhibiting knobby substructures.

\section{SEM of meiosis I and II}

SEM investigation of zygotene allows discrimination of single chromosomes and "forked" chromosomes which represent synapsed and non-synapsed homologs that can be readily discriminated from superposed chromosomes (Fig. 1a). Telomeric regions could not be observed. Higher magnification reveals that chromosome surfaces are composed of coiled and parallelarranged fibers. Synapsis of two homologs is characterized by fibrillar elements connecting both homologs (Fig. 1b). "Synaptic forks" become visible, showing chromatid strands of variable diameters from 50 to $100 \mathrm{~nm}$ which change to their homologous counterpart (Fig. 1c). In contrast to LM investigations where pachytene chromosomes appear completely paired, SEM micrographs typically show short segments where synapsis is incomplete (Fig. 2). Fibrillar threads of about 40-100 nm - not detectable with light microscopy - are frequently observed connecting non-homologous chromosomes (Figs. 2 and 3). When homologs are folded back, similar connections become evident between interstitial sites within one paired homolog (Fig. 3). In pachytene, homologs are so intimately paired that discrimination of individual homologs or individual chromatids is not possible. The compactness of the chromosome axis prevents insight into the synaptonemal complex. Only few (1-4) telomeres become visible which are typically club-shaped. Chromosomes in early diplotene are reminiscent to those of pachytene, as they are still paired over long distances, but show an increased diameter (Fig. 4). Chromosome surface is granular; 
only few parallel fibers could be observed. Fibrillar threads between non-homologous chromosomes are persistent (Fig. 5). In late diplotene, homologs - which are irregularly shaped - lie in intimate contact and are twisted around each other (Fig. 6). In contrast to earlier stages homologous chromosomes can then be discriminated at their telomeric regions (Fig. 6). Chromatin protuberances emanate from the chromosome surface; some of them typically "bridging" over long distances between nonhomologs (Fig. 6) as well as between homologs (Fig. 7). Primary and secondary constrictions were not observed. In diakinesis, when homologs separate at interstitial sites, fibrillar connections are frequently observed when chromosomes dissociate (Fig. 8). Chiasmata become visible at telomeric regions (Fig. 8), characterized by chromatid strands of about $100-300 \mathrm{~nm}$ in diameter traversing to their homologous counterpart (Fig. 9). During transition to metaphase I chromosomal morphology changes remarkably: Chromosomes are highly condensed and fused at both telomeres (Fig. 10a). Even at this stage of maximum condensation, neither primary nor secondary constrictions are visible. The putative centromere can be identified by chromatin strands emanating from the kinetochore region (Fig. 10a, b). At higher magnification the fibrillar character of these strands becomes visible (Fig. 10b). In anaphase I and telophase I chromosomes remain fully condensed and are typically U-shaped compared to V-shaped chromosomes in mitosis. Decondensation in interkinesis is incomplete and distinguishable chromosomal segments can occasionally be observed (data not shown). In prophase II chromatids are widely separated with the exception of a pericentric region (Fig. 11). Compared to mitosis, chromatid arms in meiosis II are "kinky" (Fig. 11) and flatten during spreading. At higher magnifications the coiled and parallel-arranged fibers become obvious (Fig. 12). In metaphase II chromosomes, which are highly compacted in length $(6-7 \mu \mathrm{m})$, are $X$-shaped without any constrictions. Occasionally, chromatid arms are fused at their telomeres (Fig. 13a). Even at high magnification they cannot be discriminated at their site of association (Fig. 13b). In anaphase I decondensation starts very early. In tetrad stage chromatin is decondensed and similar in appearance to interphase (not shown).

\section{Controlled loosening of chromosomes with proteinase $K$}

Controlled loosening of glutaraldehyde fixed chromosomes with proteinase $\mathrm{K}$ treatment (Wanner and Formanek, 2000) was successfully applied to meiotic chromosomes providing insight into chromatin architecture even of highly compact chromosomes. In early leptotene only short chromosomal segments are evident, which are connected within a fibrillar network (Fig. 14a). At higher magnifications the arrangement of the 30-nm fibers (loops, coils, knobs, parallel fibers) becomes obvious (Fig. 14b). In pachytene, homologs remain paired after proteinase K treatment (Fig. 15a) with exception of few small segments which are non-synapsed (Fig. 15b). At the site of synapsis, transversal elements (which are described as core element of the SC by TEM analysis of pachytene spreads) were not observed, although the depth of view would be sufficient (Fig. 15d). A chromosomal "segmentation" becomes evident: chromomere-like structures alternate with longitudinally arranged fibers (Fig. 15b). Although these fibers are in the range of $30 \mathrm{~nm}$ they vary in size from 25 to $45 \mathrm{~nm}$ and form secondary structures as observed in early leptotene (Fig. 15c). Besides $30-\mathrm{nm}$ fibers, a minor fraction of $15-\mathrm{nm}$ fibers, mainly orientated in parallel to the chromosome axis, are observed (Fig. 15d). Discrimination of homologs is not possible as they form a homogeneous network (Fig. 15c, d). In metaphase I chromosome surface is fibrillar (Fig. 16a). The centromeric region is characterized by bundles of parallel fibers emanating from the (putative) kinetochore region (Fig. 16b).

\section{DNA staining with platinum blue}

Simultaneous detection of SE and BSE signals provides a good correlation between topographic information and DNA distribution. At higher magnifications the comparison of both image types allows discrimination between chromatin fibers (= protein + DNA) and fibrillar substructures which consist of protein alone. Investigations of fibrillar connections, typically observed between non-homologs in zygotene (Fig. 1a) and pachytene (Fig. 2) stain well with platinum blue (Figs. 17 and 18 ), which proves that they contain DNA. In pachytene chromosome staining becomes more uniform with higher signal intensity along the chromosome (Fig. 18a). Telomeric regions, which can be hardly detected by topographic images, show bright BSE signals, which facilitate their identification (Fig. 18a). Some telomeric regions are associated to interstitial segments by fibrillar elements which stain with platinum blue (Fig. 18b). Structural differences visible by topographic images are more pronounced in the BSE presentations, which display changes of DNA content by changes in brightness (Fig. 18b and 19). Homologous chromosomes are intimately paired in regions with coiled fibers, which correlate with high signal intensity in BSE (Fig. 19). Short interspersed segments of approximately $100 \mathrm{~nm}$ in length, which are characterized to be less in diameter and to be dominantly organized by longitudinally arranged fibers in SE micrographs, show less signal intensity in BSE (Fig. 19). In diplotene chromosomes are irregularly shaped in SE. DNA distribution proves that there is no strict correlation between chromosome topography and signal intensity. Segments, which are prominent in the SE image, can show only a very weak BSE signal (Fig. 20a). Homologs can be hardly discriminated in SE and BSE with exception of some telomeric regions, which are typically clubshaped with an intense BSE signal (Fig. 20a). When diplotene spreads are treated with proteinase $\mathrm{K}$, chromosomes loosen. At higher magnifications a complex fibrillar network of aggregated and longitudinally arranged fibers becomes evident in the BSE image. Comparison with the topographic image reveals that some parallel fibers contain DNA while others do not (Fig. 20b). In metaphase I bivalents are characteristically attached at distal regions where no discrimination of individual telomeres is possible, neither in SE nor BSE (Fig. 21). The topographic image typically shows emanating "tips" at the centromere region that stain intensively with platinum blue, which suggests that these tips are DNA rich and part of the kinetochore but not of the spindle fiber apparatus. In prophase II chromosomes are irregularly shaped. DNA distribution reveals a good correlation between topography and BSE signal intensity (Fig. 22). In prophase II, multiple interconnecting fibers, which are typically observed between sister and non-sister chromatids, contain DNA (Fig. 22). 

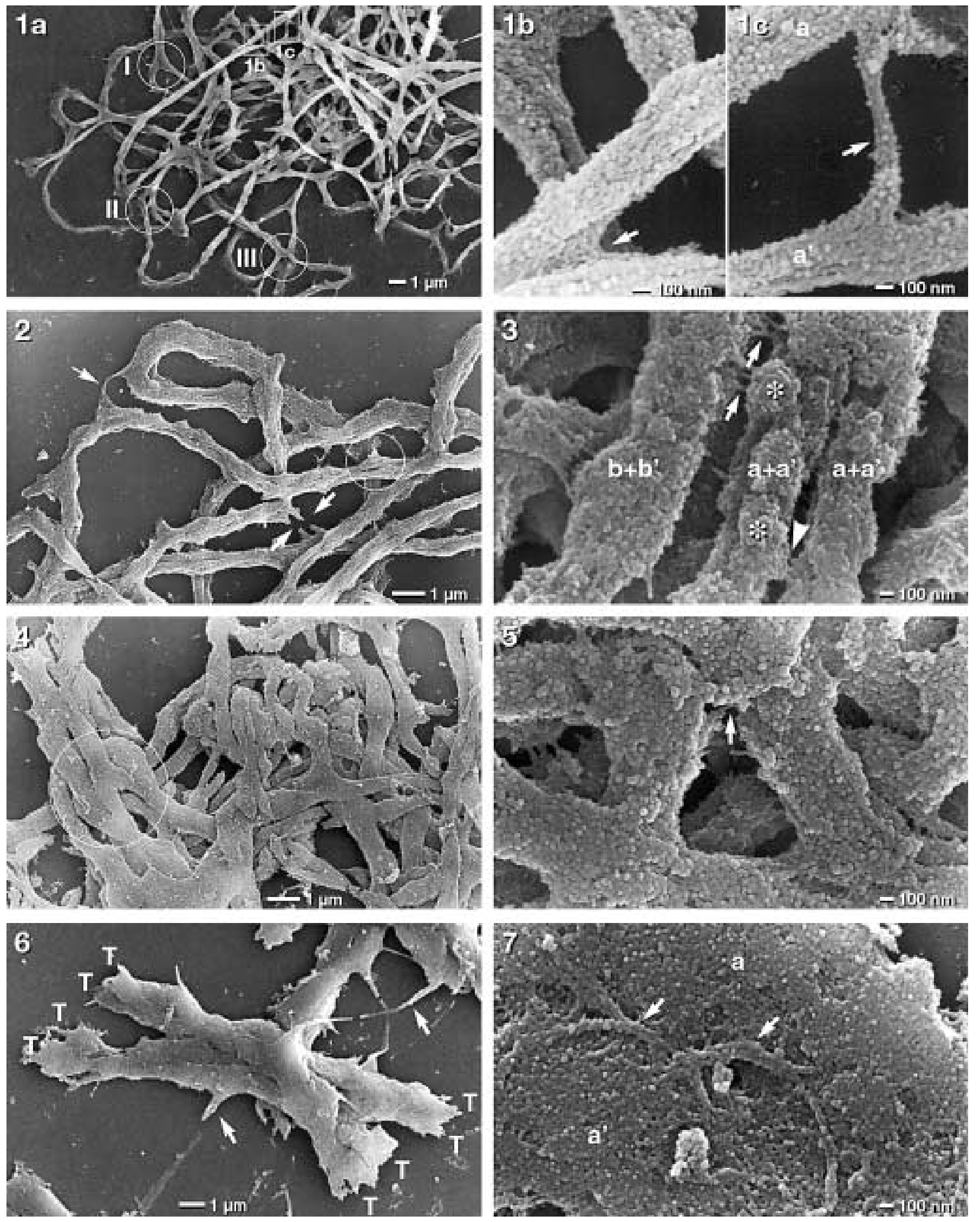

(For legends see page 150.) 

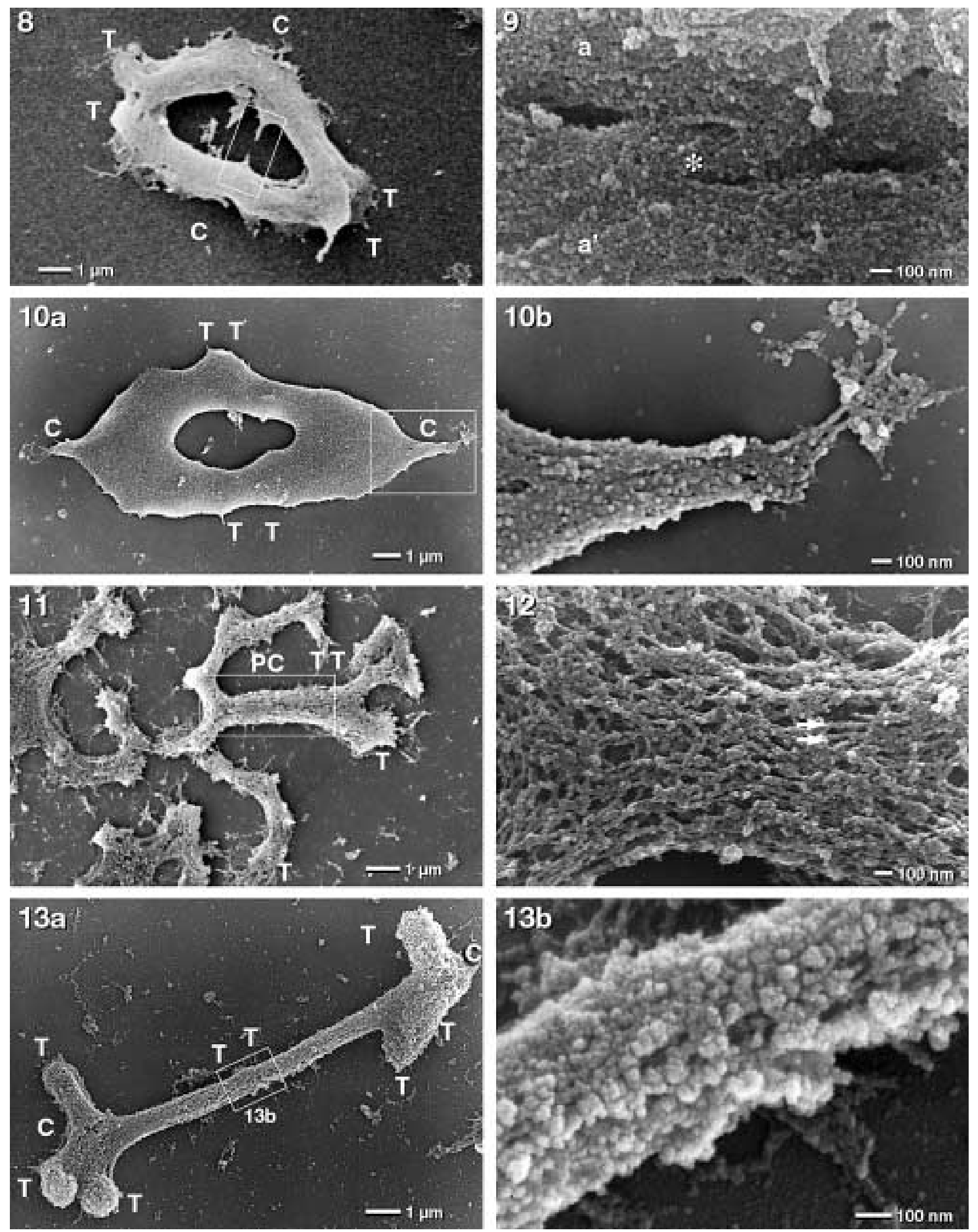

(For legends see page 150.)

Cytogenet Genome Res 105:145-156 (2004) 


\section{Discussion}

\section{Chromatin organization in early stage of meiosis}

High-resolution SEM has proven to be a powerful tool for investigations of chromosome arrangement and chromatin organization of all stages of meiosis I and II. It was shown that chromatin in meiosis I and II shares some common features in ultrastuctural details with that in mitosis; organization into fibers of $10-15 \mathrm{~nm}$ and $30 \mathrm{~nm}$ in diameter, which are arranged predominantly coiled or in parallel in early prophases. This indicates a universal arrangement of chromatin into chromosomes. The parallel fibers are found in two classes: $30-\mathrm{nm}$ fibers, interpreted as solenoids, which stain with platinum blue (see Fig. 20b), and fibers of about 15-30 nm in diameter, which do not stain and are interpreted as "matrix fibers" and are probably involved in the "dynamics" of chromosome condensation (Wanner and Formanek, 2000). However, there is a significant ultrastructural difference: parallel fibers and chromomeres are observed less frequently during meiosis compared to mitosis, which can be explained by a significantly higher compaction of meiotic chromatin (about twice as much in metaphase) that obscures these elements (Zoller et al., 2004).

Structural analysis of chromatin arrangement in meiotic chromosomes is limited by extreme compactness of meiotic chromosomes and by the lack of distinction between DNA and protein. This problem was successfully overcome by application of specific staining of DNA with "platinum blue" in combination with proteinase $\mathrm{K}$ treatment (Wanner and Formanek,

Figs. 1-13. SEM analysis of meiotic chromosomes of rye (Secale cereale; homologous chromosomes are labeled $\mathrm{a}+\mathrm{a}^{\prime}$, non-homologous chromosomes are labeled $\mathrm{b}+\mathrm{b}^{\prime} ; \mathrm{C}=$ centromeres, $\mathrm{T}=$ telomeres). In zygotene chromosomes are disordered (1a); a synapsed homolog (1, circle I) can be discriminated from attached (1, circle II) or superposed chromososomes (1, circle III). A fibrillar network characterizes synapsis of homologs (1b, arrow). Part of a chromatid of one homolog (1c) changes to its homologous counterpart (1c arrow). In pachytene, homologs are intimitately paired with exception of few, non-synapsed segments (circle). Chromatin threads become evident between non-homologs (2). Even at higher magnification, fibers connecting nonhomologs (2, arrows and $\mathbf{3}$, arrows) as well as interstitial sites of one homolog are visible $\left(a+a^{\prime} / a+a^{\prime}\right)(\mathbf{3}$, arrowhead). In early diplotene, interstitial connections between homologous and non-homologous chromosomes are still visible at low (4, circle) and higher magnification (5, arrow). In late diplotene, homologs are irregularly shaped and can hardly be discriminated at their telomeric regions $(\mathbf{6}, \mathrm{T})$. Fibers, emanating from the surface, connecting nonhomologous chromosomes (6, arrows) or homologs which are still associated (7, arrows). In diakinesis, chiasmata become visible at telomeric regions (8, T). At interstitial sites threads between bivalents are frequently observed ( 8 boxed area). Chiasmata are characterized by chromatid strands which change from one homolog to its counterpart (9, asterisk). In metaphase I, neither primary nor secondary constrictions are detectable, when telomeres of bilavents are fused (10a). At the centromeric region a putative kinetochore becomes visible by "pulled" tips (10a, boxed area), that show fibrillar character at higher magnification (10b). In prophase II, chromosomes are "kinky", when sister chromatids are separated (11) with exception of a pericentric domain $(\mathbf{1 1}$, boxed area $=$ PC). During prophase II, coiled fibers dominate with short segments of parallel fibers (12, arrows). In metaphase II, chromosomes are highly condensed. Chromatids show a structural continuity at (putative) points of translocation (13a, boxed area), even at higher magnification (13b). Chromatin fibers are rather granular.
2000). Controlled loosening of chromatin (treatment of glutaraldehyde fixed chromosomes with proteinase K) proves that meiotic chromosomes - even in condensed stages - are indeed composed of the same substructures as mitotic chromosomes (see Figs. 14, 15 and 20b). By detection of back-scattered electrons (BSE) after platinum blue staining it is obvious that DNA distribution along the chromosome arms varies to a greater degree than in mitosis (unpublished data). No evidence was found for radial loop and sequential helical coiling models as proposed for meiotic chromosomes even at low stages of condensation (Stack and Anderson, 2001). Chromosome architecture in mitosis is composed as a larger-scale structural organization (Belmont et al., 1987) mainly due to irregular aggregation of subdomains (chromomeres) alternating with longitudinal fibers (see Fig. 20b) as proposed by the dynamic matrix model (Wanner and Formanek, 2000). This organization can also be applied in principle to early stages of meiosis I. For meiosis II it has been shown for the first time that chromatin organization of all stages is different from both meiosis I and mitosis, which could reflect cell cycle specific modulation (Zoller et al., 2004).

\section{Alignment and synapsis}

Leptotene chromosomes are organized in an interphase-like stage, which probably reflects an open chromatin conformation, accessible for active transcription and recognition of homologs (Wu and Lichten, 1994). In zygotene, chromosomes obviously undergo structural reorganization, which becomes visible by chromomeres that are expressed less and orientated longitudinally in contrast to mitotic stages, where knobby chromomeres are characteristically found at all stages of condensation (Martin et al., 1996; Wanner and Formanek, 2000; Zoller et al., 2004). This finding is in good agreement with LM data of maize demonstrating morphological changes prior to synapsis that causes an elongation of "knob heterochromatin" and an increase in "surface complexity" (Dawe et al., 1994). For Lilium longiflorum it was also shown by three-dimensional light microscopy that heterochromatin segments become elongated during zygotene while the total chromosome volume decreases down to $50 \%$ (Dawe et al., 1994), which may reflect the high compaction of chromatin, confirmed by staining with platinum blue in early stages of prophase.

Figs. 14-16. Controlled denaturation after proteinase $K$ treatment in meiosis I of rye (Secale cereale). In early leptotene, the chromosomes form a fibrillar network where individual chromosome strands can be followed only segmentally (14a). At higher magnifications a fibrillar network predominantly of coiled and short parallel fibers becomes visible (14b: detail from 14a). In pachytene, the homologous chromosomes remain synapsed (15a). Homologs cannot be discriminated even at higher magnification with exception of non-synapsed regions (15b, boxed area; compare with detail 15d). Chromomere-like substructures (15b, asterisk) and segments with parallel fibers (15c and d, arrows) alternate. At the site of synapsis a complex network becomes visible (15d). In metaphase I, bivalents are still connected at their telomeric regions (16a). Parallel fibers are visible at the chromatin "tips" at the centromeric region $(\mathbf{1 6 b})$. 

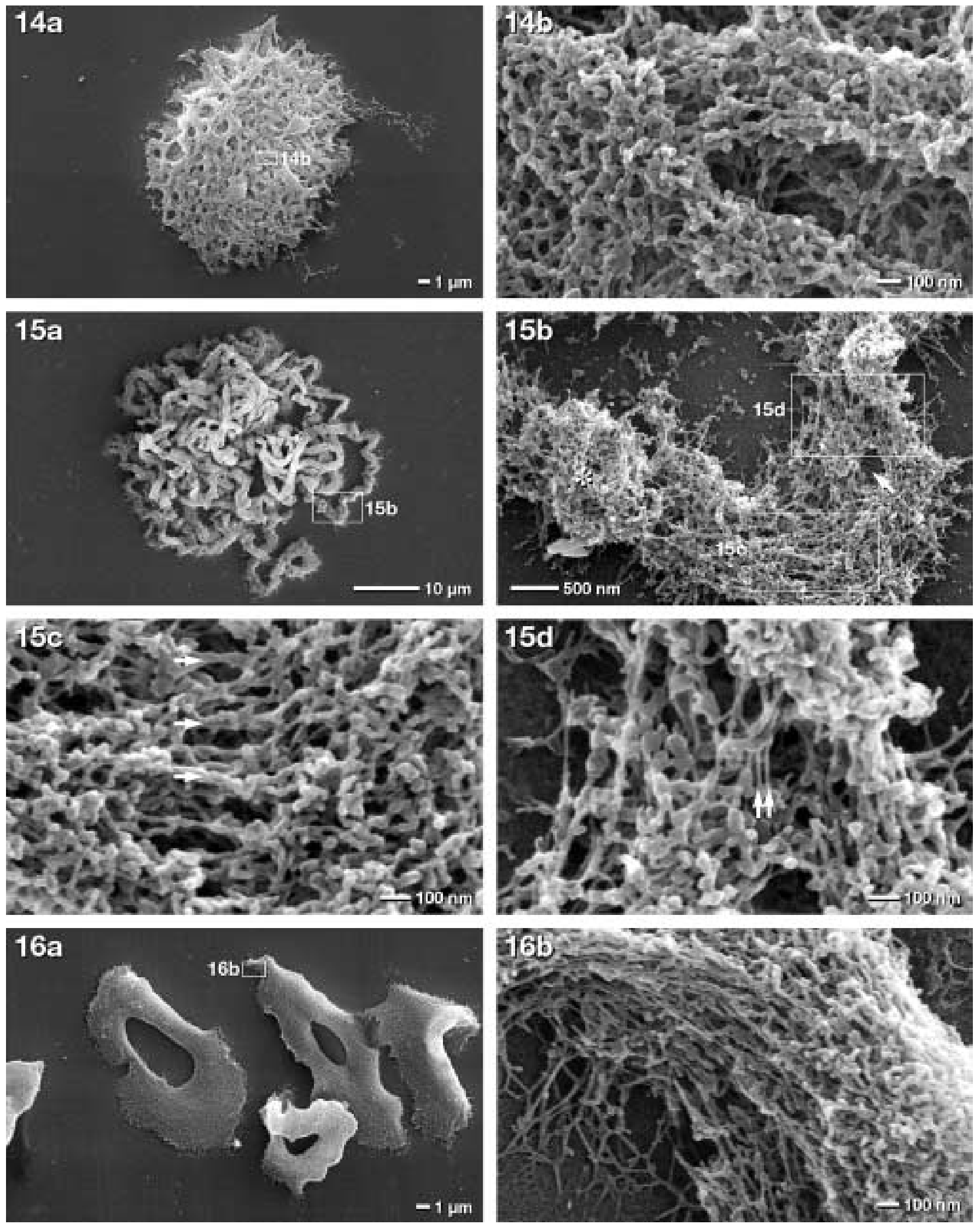

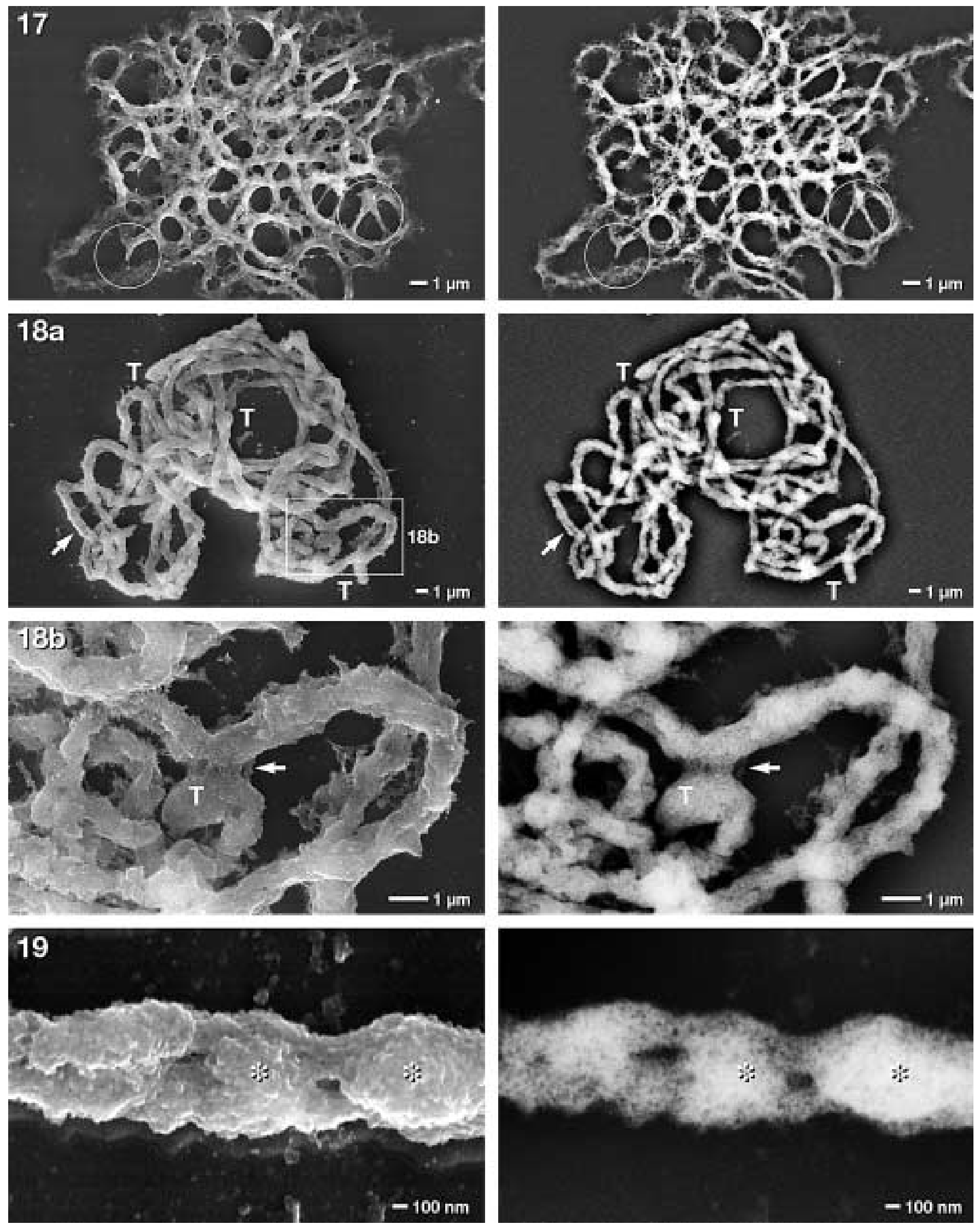

(For legends see page 154.) 

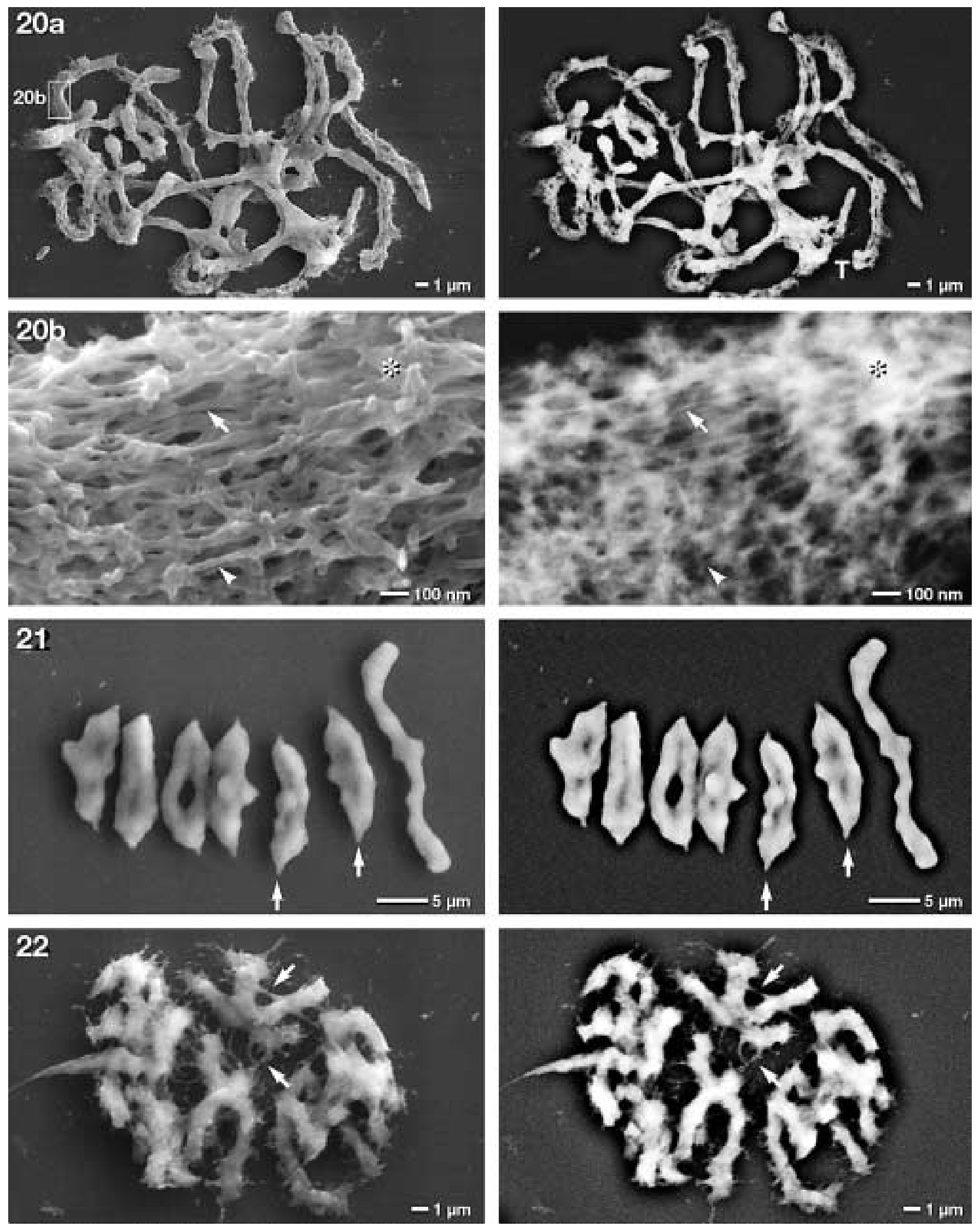

(For legends see page 154.) 
The reciprocal alignment and synapsis of homologs are proposed as segmental separation of chromatid strands that traverse to their homologous counterpart (Roeder, 1997) - but ultrastructural data were not available. These LM investigations can now be supported by the present ultrastructural analysis, which shows that there are indeed visible transitions of individual chromatids to their homologous counterpart (see Fig. 1c). Fibrillar elements that become evident at the site of synapsis (see Fig. 1b) may reflect the first visible event of homolog recognition, as described by von Wettstein et al. (1984). These connections were postulated either as proteinprotein (Kleckner and Weiner, 1993) or DNA-DNA interactions (Weiner and Kleckner, 1994; Sybenga, 1999). Their functions have been reviewed by Radman and Wagner (1993), Riggs (1997) and Sybenga (1999), although it cannot be excluded that these connections are indications of early recombination events and may represent part of the SC (Padmore et al., 1991; Scherthan, 2001).

\section{Homologous recombination}

For reciprocal recombination a 4-strand model was postulated from theoretical considerations, without ultrastructural evidence, for genetic exchange by crossing-over and Holiday junction. With SEM it could be shown for pachytene that chromosomes and chromatids are not discernible when associated to a single chromosome strand, resulting in a more or less homogeneous filament. Topographic information suggests that potential sites of recombination are characterized by a striking structural continuity of the chromosomal filament and - shown by staining for DNA - a continuity of chromatin fibers (see Figs. 18a, b and 19). It is therefore not surprising that the diameter of paired homologs is reduced - compared with the sum of diameters of individual chromosomes (Zoller et al., 2004). This tight association may influence compaction and arrangement of chromatin within homologs and would explain why the elements of the SC are not visible.

Figs. 17-22. DNA distribution after staining with platinum blue and detection with back scattered electrons (BSE) and chromosome topography with secondary electrons (SE) in meiosis I and II of rye (Secale cereale). SE images (left); BSE images (right). In zygotene, DNA distribution (BSE) shows good correlation with chromosome topography (SE) (17). Fibrillar connections between non-homologous chromosomes stain with platinum blue (17, circles). In pachytene, distribution of DNA is rather inhomogeneous. Even at low magnifications non-synapsed segments are visible in the BSE image in contrast to the SE image (18a, arrows). Few club-shaped telomeres show brighter signals due to a higher DNA content (18a, T). Connections of telomeric regions to interstitial sites stain positively with platinum blue $\mathbf{( 1 8 b}$, arrows). Higher magnification reveals that homologs are completely synapsed within chromomere-like segments (19, asterisks), while non-synapsed segments are characterized by longitudinal fibers (19). In diakinesis, individual homologs are hardly discernible with exception of their telomeric regions which can be discriminated by high signal intensity $(\mathbf{2 0 a}, \mathrm{T})$. When chromosomes in diakinesis are treated with proteinase $\mathrm{K}$, their chromatin loosens giving insight into a fibrillar network (20b). Most parallel fibers are stained (20b, arrows), some are not stained (20b, arrowheads). In metaphase I, chromosomes stain rather uniformly for DNA (21). Chromatin "tips" at the centromeric regions contain DNA; although they are tiny, they give a bright BSE-signal (21, arrows). In prophase II, individual chromatid arms are "kinky"; they are typically interconnected by chromatin threads (22, arrows).
Chromosome recombination and formation of SC is assumed to be complete in pachytene (Schmekel and Daneholt, 1995). From SEM data this does not seem to be the case, as synapsed and non-synapsed segments alternate during pachytene (see Figs. 2 and 19), which are not detectable in LM due to low resolution. It has been postulated that pairing of chromosomes can be influenced by the level of homology and amount of repetitive sequences (Karpen et al., 1996; Buckler et al., 1999). These findings are supported by our observation that synapsis is complete in segments with coiled fibers, which can be attributed to heterochromatic segments, and is incomplete in segments with longitudinal fibers, which can be assigned to euchromatic domains due to their smaller diameter (see Fig. 19). There are two major explanations for the discrepancy between LM and SEM data: (i) synapsis, in general, is found less in euchromatic regions than in heterochromatic regions which are more likely to present greater homology; (ii) synapsis and the formation of the SC are dynamic and develop segmentally along chromosomes. These considerations are supported by comparative analysis of meiosis in yeast and higher plants which shows differences in initiation of synapsis, formation of SCs as well as in the frequency of genetic exchange of homologous chromosomes (Padmore et al., 1991).

\section{Non-homologous interactions}

Homologous recombination is the primary mechanism for genetic diversity influenced by natural selection. It has been shown for rye with molecular methods that non-homologous recombination occurs occasionally without formation of chiasmata (Gill et al., 1997). Numerous small chromatin interconnecting threads are observed between dividing homologs and non-homologs (see Figs. 2-8) from pachytene to diakinesis, which might be structural evidence for molecular data of nonreciprocal gene transfer and gene conversion (Carpenter, 1987). For meiosis of yeast it was estimated that non-reciprocal gene transfer occurs at a frequency similar to that observed between allelic genes (Jinks-Robertson and Petes, 1985); this process is discussed to be linked with genomic rearrangements playing a significant role in karyotypic evolution and speciation for primates (Stankewics and Lupski, 2002). Non-reciprocal gene transfer is also described for B chromosomes and for polyploid genome constitutions in rye (Santos et al., 1993, 1995). In this context we interpret multiple chromatin connections between non-homologous chromosomes (see Figs. 2-6, 17, 18b) as structures facilitating gene transfer and potential sites of chromatin exchange. In addition, we assume that intrachromosomal connections, frequently observed within paired homologs (see Fig. 3), may be an indication for intrachromosomal recombination which seems to occur predominantly between multiple tandem units of closely linked DNA repeats (Peterhans et al., 1990). These unequal exchanges are discussed to represent the primary mechanism of meiotic instability of genes - one of several possible mechanisms for rearrangement of satellite DNA in plant chromosomes (Fincham and Sastry, 1974; reviewed by Federoff, 1984) - and are essential for the increase and variation of DNA amount among various grass species (Vershinin et al., 1995; Flavell, 1980). 


\section{Chiasmato}

It is widely accepted that chiasmata are visible structures of crossing-over events (Jones and Rees, 1964). Assuming that each of the four chromatids has the same diameter, only "strands" (parts of a chromatid) between one of the non-sister chromatids would be exchanged (see Fig. 9). During transition to metaphase I these chiasmata become manifested as telomeric fusions as shown by DNA staining (see Fig. 21) and treatment with proteinase $\mathrm{K}$ (see Fig. 16a). From this point of view a "punctual agglutination" by residual SC proteins (Zickler, 1977; Holm and Rassmussen, 1977) is rather unlikely.

\section{NORs and centromeres}

Although primary and secondary constrictions are the most important morphological features for characterization of mitotic chromosomes it is surprising that meiotic chromosomes lack constrictions. This feature could be explained by the rearrangement of centromeric regions of both sister chromatids to form one single, functional centromere and, respectively, kinetochore. CENP-E protein in mammalia shows that kinetochores in meiosis I are apparently fused (Lee et al., 2000). Chromatin protuberances being expelled from the putative centromeric regions of meiotic chromosomes can be regarded as a visible effect of increased physical forces onto meiotic metaphase chromosomes that are subject to a stronger force - due to the fused telomeric regions when homologs are pulled to the opposite poles (see Figs. 10, 16a, 21). This finding also correlates with SEM investigations of kinetochores of Tradescantia, showing protrusions at the centromeres (Inaga et al., 2000).

Further studies applying these new SEM techniques in combination with immunolocalization of recombination-specific proteins and in situ hybridization will open new avenues of investigation and contribute to a deeper understanding of structural organization and changes of chromosomes in meiosis.

\section{Acknowledgements}

We thank Dr. B. Schinkel, Lochow-Petkus, for providing seeds of the rye variety "Sorom". The authors gratefully acknowledge the excellent technical assistance of Claudia Nickel and Elizabeth Schroeder-Reiter for proof-reading the manuscript.

\section{References}

Barlow AL: Electron microscopic in situ hybridization (EMISH) against synaptonemal complex-associated chromatin. Chromosome Res 4:248-249 (1996).

Barlow AL, Jenkins G, ap Gwynn I: Scanning electron microscopy of synaptonemal complexes. Chromosome Res 1:9-13 (1993)

Bass HW, Riera-Lizarazu O, Ananiev EV, Bordoli SJ, Rines HW, Phillips RL, Sedat JW, Agard DA Cande WZ: Evidence for the coincident initiation of homolog pairing and synapsis during the telomere-clustering (bouquet) stage of meiotic prophase. J Cell Sci 113:1033-1042 (2000).

Belmont AS, Sedat JW, Agard DA: A three-dimensional approach to mitotic chromosome structure: Evidence for a complex hierarchical organization. J Cell Biol 105:77-92 (1987).

Buckler ES 4th, Phelps-Durr TL, Buckler CS, Dawe RK, Doebley JF, Holtsford TP: Meiotic drive of chromosomal knobs reshaped the maize genome. Genetics 153:415-426 (1999).

Carpenter AT: Gene conversions, recombination nodules, meiotic recombination and chiasmata. Bioessays 6:32-236 (1987).

Crackower MA, Kolas NK, Noguchi J, Sarao R, Kikuchi K, Kaneko H, Kobayashi E, Kawai Y, Kozieradzki I, Landers $\mathrm{R}$, Mo R, Hui CC, Nieves E, Cohen PE, Osborne LR, Wada T, Kunieda T, Moens PB, Penninger JB: Essential role of Fkbp6 in male fertility and homologous chromosome pairing in meiosis. Science 300:1291-1295 (2003).

Dawe RK, Sedat JW, Agard DA, Cande WZ: Meiotic chromosome pairing in maize is associated with a novel chromatin organization. Cell 76:901-912 (1994).

Federoff NV: Transposable elements in maize. Sci Am 250:64-74 (1984).

Fincham JRS, Sastry GRK: Controlling elements in maize. Annu Rev Genet 8:15-50 (1974).

Flavell RB: Molecular changes in chromosomal DNA organisation and origins of phenotypic variation. Chromosomes Today 7:42-54 (1980).
Gill BS, Gill KS, Friebe B, Endo TR: Expanding genetic maps: reevaluation of the relationship between chiasmata and crossovers, in Henriques-Gil N, Parker JS, Puertas MJ (eds): Chromosomes Today, vol 12, pp 283-298 (Chapman \& Hall, London 1997).

Hanks SK, Gollin SM, Rao PN, Wray W, Hittelman WN: Cell cycle-specific changes in the ultrastructural organization of prematurely condensed chromosomes. Chromosoma 88:333-342 (1983).

Holm P, Rassmussen S: Human meiosis I. The human pachytene karyotype analyzed by three dimensional reconstruction of the synaptonemal complex. Carlsberg Res Commun 42:283-323 (1977).

Inaga S, Naguro T, Kameie T, Iino A: Three dimensional ultrastructure of in situ chromosomes and kinetochores of Tradescantia reflexa anther cells by scanning electron microscopy. 2. Whole mounted chromosomes and kinetochores of pollen mother cells and tapetal cells. Chromosome Sci 4:11-20 (2000)

Inaga S, Tanaka K, Jino A: Three-dimensional helical coiling structures and band patterns of hydrous metaphase chromosomes observed by low vacuum scanning electron microscopy. Arch Histol Cytol 65:415-423 (2002)

Iwano M, Fukui K, Takaichi S, Isogai A: A globular and fibrous structure in barley chromosomes revealed by high-resolution scanning electron microscopy. Chromosome Res 5:341-349 (1997).

Jinks-Robertson S, Petes TD: High-frequency meiotic gene conversion between repeated genes on nonhomologous chromosomes in yeast. Proc Natl Acad Sci USA 82:3350-3354 (1985)

Jones GH, Rees H: Control of chromosome behaviour in rye VII: The distribution of chiasmata within pollen mother cells. Heredity 19:719-730 (1964).

Karpen GH, Le MH, Le H: Centric heterochromatin and the efficiency of achiasmate disjunction in Drosophila female meiosis. Science 273:118-122 (1996).

Kleckner N, Weiner BM: Potential advantages of unstable interactions for pairing of chromosomes in meiotic somatic and premeiotic cells. Cold Spring Harb Symp Quant Biol 58:553-565 (1993).
Lee J, Miyano T, Dai Y, Wooding P, Yen TJ, Moor RM: Specific regulation of CENP-E and kinetochores during meiosis I/meiosis II transition in pig oocytes. Mol Reprod Dev 56:51-62 (2000).

Martin R, Busch R, Herrmann RG, Wanner G: Efficient preparation of plant chromosomes for highresolution scanning electron microscopy. Chromosome Res 2:411-415 (1994).

Martin R, Busch W, Herrmann RG, Wanner G: Changes in chromosomal ultrastructure during the cell cycle. Chromosome Res 4:288-294 (1996).

Murayama K: Stereoscopic scanning electron microscopy of the chromosomes in Vicia faba (Broad Beans). J Ultrastruct Res 82:322-326 (1983).

Nagai S, Inoue T, Iino A: Fibrous structures of human chromosomes observed by scanning electron $\mathrm{mi}-$ croscopy. Cytobios 34:35-43 (1982).

Olins AL, Olins DE: Spheroid chromatin units (v bodies). Science 122:330-332 (1974).

Padmore R, Cao L, Kleckner N: Temporal comparison of recombination and synaptonemal complex formation during meiosis in $S$. cerevisiae. Cell 66:1239-1256 (1991).

Peterhans A, Schlupmann H, Basse C, Paszkowski J Intrachromosomal recombination in plants. EMBO J 9:3437-3445 (1990).

Radman M, Wagner R: Mismatch recognition in chromosomal interactions and speciation. Chromosoma 102:369-373 (1993).

Reznik NA, Yampol GP, Kiseleva EV, Khristolyubova NB, Gruzdev AD: Functional and structural units in the chromomere. Genetica 83:293-299 (1991).

Riggs CD: Meiotin-1: the meiosis readiness factor? Bioessays 19:925-931 (1997).

Roeder GS: Meiotic chromosomes: it takes two to tango. Genes Dev 20:2600-2621 (1997).

Santos JL, Jimenez MM, Diez M: Synaptic patterns of rye B chromosomes. I: The standard type. Chromosome Res 1:145-152 (1993).

Santos JL, Jimenez MM, Diez M: Synaptic patterns of rye B chromosomes. IV. The B isochromosomes. Heredity 74:100-107 (1995). 
Scherthan H: A bouquet makes ends meet. Nat Rev Mol Cell Biol 2:621-627 (2001).

Scherthan H, Bahler J, Kohli J: Dynamics of chromosome organization and pairing during meiotic prophase in fission yeast. J Cell Biol 127:273-285 (1994).

Schmekel K, Daneholt B: The central region of the synaptonemal complex revealed in three dimensions. Trends Cell Biol 5:239-242 (1995).

Stack SM, Anderson LK: A model for chromosome structure during the mitotic and meiotic cell cycles. Chromosome Res 9:175-198 (2001).

Stankewics P, Lupski JR: Molecular-evolutionary mechanisms for genomic disorders. Curr Opin Genet Dev 12:312-319 (2002)

Sumner AT: Electron microscopy of the parameres formed by the centromeric heterochromatin of human chromosome 9 at pachytene. Chromosoma 94:199-204 (1986)

Sybenga J: What makes homologous chromosomes find each other in meiosis? A review and a hypothesis. Chromosoma 108:209-219 (1999).

Sym M, Engebrecht JA, Roeder GS: ZIP1 is a synaptonemal complex protein required for meiotic chromosome synapsis. Cell 72:365-378 (1993).
Thoma F, Koller T, Klug A: Involvement of histone H1 in the organization of the nucleosome, and all the salt-dependent substructures of chromatin. J Cell Biol 83:408- 427 (1979).

Utsumi KR: Scanning electron microscopy of Giemsastained chromosomes and surface-spread chromosomes. Chromosoma 86:683-702 (1982).

Utsumi KR: A Scanning electron microscopy study of chromosomes and nuclei. Scan Electron Microsc III:1121-1132 (1985)

Vazquez J, Belmont AS, Sedat JW: The dynamics of homologous chromosome pairing during male Drosophila meiosis. Curr Biol 12:1473-1483 (2002).

Vershinin AV, Schwarzacher T, Heslop-Harrison JS: The large-scale genomic organization of repetitive DNA families at the telomeres of rye chromosomes. Plant Cell 7:1823-1833 (1995).

Wanner G, Formanek H: Imaging of DNA in human and plant chromosomes by high-resolution scanning electron microscopy. Chromosome Res 3:368-374 (1995).

Wanner G, Formanek H: A new chromosome model. J Struct Biol 132:147-161 (2000).
Wanner G, Formanek H, Martin R, Hermann RG: High resolution scanning electron microscopy of plant chromosomes. Chromosoma 100:103-109 (1991).

Weiner BM, Kleckner N: Chromosome pairing via multiple interstitial interactions before and during meiosis in yeast. Cell 77:977-991 (1994).

von Wettstein D, Rasmussen SW, Holm PB: The synaptonemal complex and genetic segregation. Symp Soc Exp Biol 38:195-231 (1984).

Woodcock CLF: Ultrastructure of inactive chromatin J Cell Biol 59:368a (1973).

Woodcock CLF, Safer JP, Stanchfield JE: Structural repeating units in chromatin. Evidence for their general occurrence. Exp Cell Res 97:101-110 (1976).

Wu TC, Lichten M: Meiosis induced double-strand break sites determined by yeast chromatin structure. Science 263:515-518 (1994)

Zickler D: Development of the synaptonemal complex and the "recombination nodules" during meiotic prophase in the seven bivalents of the fungus Sordaria macrospora Ruersw. Chromosoma 61:289316 (1977).

Zoller JF, Herrmann RG, Wanner G: Chromosome condensation in mitosis and meiosis of rye (Secale cereale L.). Cytogenet Genome Res 105:134-144 (2004). 